\title{
https://helda.helsinki.fi
}

\section{Pattern Discovery in Colored Strings}

\section{Lipták, Zsuzsanna}

Schloss Dagstuhl - Leibniz-Zentrum für Informatik 2020-06-01

Lipták , Z , Puglisi , S J \& Rossi , M 2020 , Pattern Discovery in Colored Strings . in S Faro \& D Cantone (eds) , 18th International Symposium on Experimental Algorithms (SEA 2020) . , 12 , Leibniz International Proceedings in Informatics, LIPIcs, vol. 160 , Schloss Dagstuhl Leibniz-Zentrum für Informatik , Dagstuhl , International Symposium on Experimental Algorithms , Catania , Italy , 16/06/2020 . https://doi.org/10.4230/LIPIcs.SEA.2020.12

http://hdl.handle.net/10138/340721

https://doi.org/10.4230/LIPIcs.SEA.2020.12

cc_by

publishedVersion

Downloaded from Helda, University of Helsinki institutional repository.

This is an electronic reprint of the original article.

This reprint may differ from the original in pagination and typographic detail.

Please cite the original version. 


\title{
Pattern Discovery in Colored Strings
}

\author{
Zsuzsanna Lipták \\ Department of Computer Science, University of Verona, Italy \\ zsuzsanna.liptak@univr.it
}

Simon J. Puglisi

Department of Computer Science, University of Helsinki, Finland

puglisi@cs.helsinki.fi

\section{Massimiliano Rossi}

Department of Computer and Information Science and Engineering, University of Florida, Gainesville, FL, USA

rossi.m@ufl.edu

\begin{abstract}
We consider the problem of identifying patterns of interest in colored strings. A colored string is a string in which each position is colored with one of a finite set of colors. Our task is to find substrings that always occur followed by the same color at the same distance. The problem is motivated by applications in embedded systems verification, in particular, assertion mining. The goal there is to automatically infer properties of the embedded system from the analysis of its simulation traces. We show that the number of interesting patterns is upper-bounded by $\mathcal{O}\left(n^{2}\right)$ where $n$ is the length of the string. We introduce a baseline algorithm with $\mathcal{O}\left(n^{2}\right)$ running time which identifies all interesting patterns for all colors in the string satisfying certain minimality conditions. When one is interested in patterns related to only one color, we provide an algorithm that identifies patterns in $\mathcal{O}\left(n^{2} \log n\right)$ time, but is faster than the first algorithm in practice, both on simulated and on real-world patterns.
\end{abstract}

2012 ACM Subject Classification Theory of computation $\rightarrow$ Design and analysis of algorithms

Keywords and phrases property testing, suffix tree, pattern mining

Digital Object Identifier 10.4230/LIPIcs.SEA.2020.12

Related Version A full version of the paper is available at https://arxiv.org/abs/2004.04858.

Supplementary Material An implementation of the algorithms is available online at https://github. com/maxrossi91/colored-strings-miner.

Funding Simon J. Puglisi: partially funded by the Academy of Finland via grant 319454 . Massimiliano Rossi: partially funded by the Ph.D. School of the University of Verona.

Acknowledgements We thank Johannes Fischer, Travis Gagie, and Ferdinando Cicalese for interesting discussions, and Alessandro Danese for providing an updated data set of traces.

\section{Introduction}

In recent years, embedded systems have become increasingly pervasive and are becoming fundamental components of everyday life. In line with this, embedded systems are required to perform increasingly demanding tasks, and in many circumstances, peoples' lives are now dependent on the correct functioning of these devices. This, in turn, has led to an increasingly complex design process for embedded systems, where a major design task is to evaluate and check the correctness of the functionality from the early stages of the development process. This functionality checking is usually done using assertions - logic formulae expressed in temporal logic such as Linear Temporal Logic (LTL) or Computation Tree Logic (CTL) that provide a way to express desirable properties of the device. Assertions are typically written by hand by the designers and it might take months to obtain a set of assertions

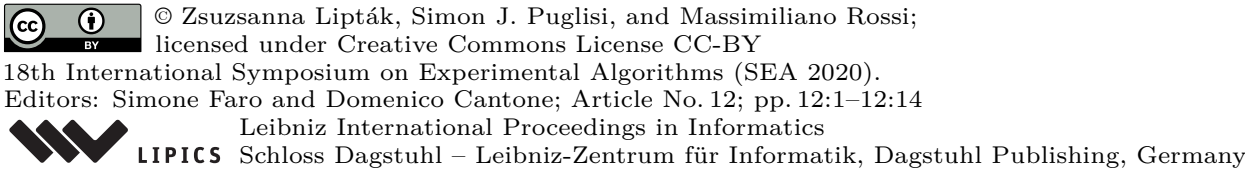


(a) Simulation trace.

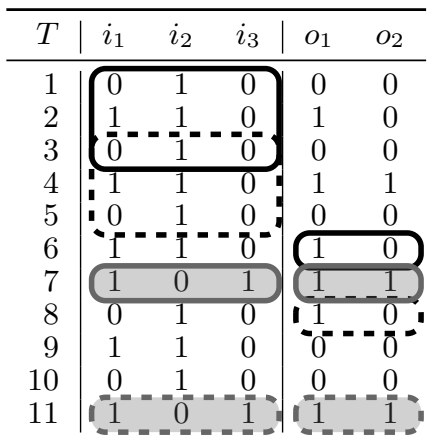

(b) Mapping of the input and output alphabet.

\begin{tabular}{ccc|cccc|c}
\multicolumn{3}{c}{ Input alphabet. } & & \multicolumn{3}{c}{ Output alphabet. } \\
\cline { 1 - 2 } \cline { 5 - 7 }$i_{1}$ & $i_{2}$ & $i_{3}$ & $\Sigma$ & & $o_{1}$ & $o_{2}$ & $\Gamma$ \\
\cline { 1 - 3 } & 1 & 0 & $\mathrm{a}$ & & 0 & $x$ \\
1 & 0 & 1 & $\mathrm{~b}$ & & 0 & $y$ \\
1 & 1 & 0 & $\mathrm{C}$ & & 1 & 1 & $z$
\end{tabular}

(c) The colored string associated with the simulation trace.

\begin{tabular}{lllllllllll}
$x$ & $y$ & $x$ & $z$ & $x$ & $y$ & $z$ & $y$ & $x$ & $x$ & $z$ \\
\cline { 1 - 2 } & $\mathrm{c}$ & $\mathrm{a}$ & $\mathrm{c}$ & $\mathrm{a}$ & $\mathrm{c}$ & $\mathrm{b}$ & $\mathrm{a}$ & $\mathrm{c}$ & $\mathrm{a}$ & $\mathrm{b}$ \\
1 & 2 & 3 & 4 & 5 & 6 & 7 & 8 & 9 & 10 & 11
\end{tabular}

Figure 1 Example of a simulation trace of a device with input ports $\mathcal{I}=\left\{i_{1}, i_{2}, i_{3}\right\}$ and output ports $\mathcal{O}=\left\{o_{1}, o_{2}\right\}$ and the associated colored string.

that is small and effective (i.e. it covers all functionalities of the device) [15]. In order to help designers with the verification process, methodologies and tools have been developed which automatically generate assertions from simulation traces of an implementation of the device $[22,32,8,7]$. The objective is to provide a small set of assertions that cover all behaviors of the device, in order to extend the basic manually-defined set of assertions.

A simulation trace can be viewed as a table that records, for every simulation instant $T$, the value assumed by the input and output ports of the device. Figure 1a shows an example of a simulation trace of a device with three input ports $\mathcal{I}=\left\{i_{1}, i_{2}, i_{3}\right\}$ and two output ports $\mathcal{O}=\left\{o_{1}, o_{2}\right\}$. An assertion is a logic formula expressed in temporal logic that must remain true in the whole trace. The simplest assertions involve only conditions occurring at the same simulation instant. In the simulation trace in Figure 1a, from the solid and dashed shaded boxes, we can assert that each time we have $i_{1}=1, i_{2}=0$, and $i_{3}=1$, then $o_{1}=1$ and $o_{2}=1$. On the other hand, we cannot assert that each time we have $i_{1}=1, i_{2}=1$, and $i_{3}=0$, then $o_{1}=1$ and $o_{2}=1$, because there is a counterexample in the simulation trace, namely at instant $T=9$, where $o_{1}=0$ and $o_{2}=0$. Note assertions need not contain all input and output variables, e.g. we can assert that $i_{1}=0$ and $i_{3}=0$ implies $o_{2}=0$.

Among all possible types of assertions that can be expressed in temporal logic, an important one is given by chains of next: sequences of consecutive input values that, when provided to the device, uniquely determine their output, after a certain number of simulation instants. For example, in the simulation trace in Figure 1a, we can assert that each time we have, for $\left(i_{1}, i_{2}, i_{3}\right)$, values $(0,1,0),(1,1,0),(0,1,0)$ in consecutive simulation instants, then, three instants later, we will see $o_{1}=1$ and $o_{2}=0$.

We model simulation traces with colored strings. A colored string is a string over an alphabet $\Sigma$, where each position is additionally assigned a color from an alphabet $\Gamma$. We will set $\Sigma$ as the set of tuples of possible values for the input ports $i_{1}, \ldots, i_{k}$ and $\Gamma$ as that of the output traces $o_{1}, \ldots, o_{r}$. The objective is to identify patterns in the string whose occurrence is always followed by the same color at some given distance.

Related Work. Pattern mining, with the seminal Apriori algorithm [1], arose from the desire to discover frequent itemsets and association rules in shopping basket data, i.e. items that were frequently bought together. Time relationhips, e.g., between entries of the database in which the basket data are stored, were later considered in so-called sequential pattern mining [2]. In sequential pattern mining, episodes are partially ordered sequences of events that appears close to each other in the sequence [25]. Given episodes of the sequence, it is 
possible to build episode rules that establish antecedent-consequent relations among episodes. Sequential pattern mining has many applications (see, e.g., $[4,21,10]$ ) and has been surveyed extensively $[28,23,16]$. Unfortunately, the above setting is not applicable to our problem, since time is given only in a relative sense, i.e., whether an event happens before (or after) another, while we need to count exactly the instants occurring between two events.

In the string mining problem [13, 12, 14, 9, 31], one aims to discover strings that appear as a substring in more than $\omega$ strings in a collection, where $\omega$ is a user-defined parameter called the support of the string. The problem has been extended to mining frequent subsequences [19] and distinguishing subsequence patterns with gap constraints [20, 27, 33, 34]. String mining, however, has only superficial similarity to the colored string problems we consider.

In assertion mining, the two existing tools, GoldMine [32] and A-Team [7], are based on data mining algorithms. In particular, GoldMine [32] extracts assertions that predicate only on one instant of the simulation trace - i.e. they do not involve any notion of time - , using decision tree based mining or association mining [1]. Furthermore, using static analysis techniques together with sequential pattern mining, it extracts temporal assertions. The tool A-Team [7], requires the user to provide the template of the temporal assertions that they want to extract. For example, in order to extract the properties of our example in Fig. 1a, one needs to provide a template stating that we want a property of the form: "a property $p_{1}$, at the next simulation instant a property $p_{2}$, at the next simulation instant a property $p_{3}$, then after three simulation instants a property $p_{4}$ ". Given a set of templates, the software, using an Apriori algorithm, extracts propositions (logic formulae containing the logical connectives $\neg, \vee$, and $\wedge$ ) from the trace. Once the propositions have been extracted, the tool generates the assertion by instantiating the extracted propositions in the templates, using a decision-tree-based algorithm to find formulas that fit in the template and are verified in the simulation trace, i.e. if the trace contains no counterexample.

Our Contribution. We introduce colored strings, and propose and analyze a pattern discovery problem on colored strings which corresponds to a useful simplification of pattern mining w.r.t. assertion mining. Given a colored string and a color as input, we must find all minimal substrings that occur followed always at a the same distance by the given color. We define this problem formally in Section 2. Although this problem is simpler than the original assertion mining problem, the solution to our problem contains all the information, possibly filtered, to recover the desired set of minimal assertions in a second stage.

We first upper bound the number of minimal patterns by $\mathcal{O}\left(n^{2}\right)$. We then describe a suffix-tree-based algorithm to find all minimal patterns, when only one color is of interest. We go on to describe several refinements to this algorithm that significantly improve its performance in practice. Finally, we consider (practically motivated) restrictions on the patterns and show that under these restrictions performance is further improved.

\section{Basics}

Let $S=S[1, n]$ be a string of length $|S|=n$ on a finite ordered alphabet $\Sigma$. $\varepsilon$ denotes the empty string of length $0 . S[i]$ denotes the $i$ 'th character of $S$ and $S[i, j]$ the substring $S[i] \cdots S[j]$, if $i \leq j$, while $S[i, j]=\varepsilon$ if $i>j$. A substring $T$ of $S$ is called proper if $T \neq S$.

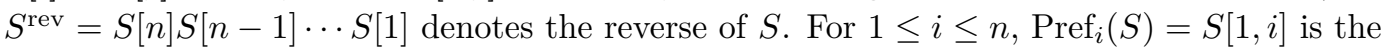
$i$ 'th prefix of $S$, and $\operatorname{Suf}_{i}(S)=S[i, n]$ is the $i$ 'th suffix of $S$. 
Colored strings. Given two finite sets $\Sigma$ (the alphabet) and $\Gamma$ (the colors), a colored string over $(\Sigma, \Gamma)$ is a string $S=S[1, n]$ over $\Sigma$ together with a coloring function $f_{S}:\{1, \ldots, n\} \rightarrow \Gamma$. We denote by $\sigma=|\Sigma|$ and $\gamma=|\Gamma|$ the number of characters resp. of colors. Given a colored string $S$ of length $n$, its reverse is denoted $S^{\text {rev }}$, and its coloring function $f_{S^{\text {rev }}}$ is defined by $f_{S_{\text {rev }}}(i)=f_{S}(n-i+1)$, for $i=1, \ldots, n$. When $S$ is clear from the context, we write $f$ for $f_{S}$ and $f^{\text {rev }}$ for $f_{S^{\text {rev }}}$.

We are interested in those substrings which are always followed by a given color $y$, at a given distance $d$. For example, let $S=$ acacacbacab, with colors $x y x z x y z y x x z$ (see Fig. 1c). Substring aca occurs 3 times in $S$, at positions 1,3, and 8 . In positions 1 and 3 it is followed by $y$ at distance 3 , while at position 8 , the corresponding position is beyond the end of $S$. This leads to the following definition.

- Definition 1 (y-good, $y$-unique, minimal). Let $S$ be a colored string over $(\Sigma, \Gamma), y \in \Gamma$ a color, $d \leq n$ a non-negative integer, and $T=T[1, m]$ a substring of $S$.

1. An occurrence $i$ of $T$ is called $y$-good with delay $d$ (or $(y, d)$-good) if $f(i+m-1+d)=y$.

2. $T$ is called $y$-unique with delay $d$ (or $(y, d)$-unique) if for every occurrence $i$ of $T, i$ is $(y, d)$-good or $i+m-1+d>n$.

3. $T$ is called minimally $(y, d)$-unique if there exists no proper substring $U$ of $T$ which is $y$-unique with delay $d^{\prime}$, for some $d^{\prime}$ s.t. $U=T[i, j]$ and $d^{\prime}=d+|T|-j$.

In the example, the occurrence of aca in position 1 is $(y, 3)$ - and $(y, 5)$-good, that in 3 is $(y, 1)$ - and $(y, 3)$-good, while that in 8 is not $(y, d)$-good for any $d$. Therefore, the substring $T=$ aca is a $(y, 3)$-unique substring of $S$, since every occurrence $i$ of aca is either $(y, 3)$-good or $i+m-1+d>n$. But aca is not minimal, since its substring ca is also $(y, 3)$-unique.

The introduction of minimally $(y, d)$-unique substrings serves to restrict the output size. Let $T=a X b$ be $(y, d)$-unique, with $a, b \in \Sigma$ and $X \in \Sigma^{*}$. We call $T$ left-minimal if $X b$ is not $(y, d)$-unique, and right-minimal if $a X$ is not $(y, d+1)$-unique. We make the following simple observations about $(y, d)$-unique substrings. (Note that 2 is a special case of 3.)

- Observation 1. Let $S \in \Sigma^{*}$ and let $T$ be a $(y, d)$-unique substring of $S$.

1. $T$ is minimal if and only if it is left- and right-minimal.

2. If $T$ is a suffix of $T^{\prime}$, then $T^{\prime}$ is also $(y, d)$-unique.

3. If $T^{\prime}=U T V$ is a superstring of $T$ such that $|V| \leq d$, then $T^{\prime}$ is $(y, d-|V|)$-unique.

We are now ready to formally state the problem treated in this paper.

- Problem 1 (Pattern Discovery Problem). Given a colored string $S$ and a color y, report all pairs $(T, d)$ such that $T$ is a minimally $(y, d)$-unique substring of $S$.

We next give an upper bound on the number of minimally $(y, d)$-unique substrings.

- Lemma 2. Given string $S$ of length $n$, the number of minimally $(y, d)$-unique substrings of $S$, over all $y \in \Gamma$ and $d=0, \ldots, n$, is $\mathcal{O}\left(n^{2}\right)$.

Suffix trees and indexed priority queue. We assume some familiarity with the suffix tree data structure, (see e.g. $[18,30,24]$ ). We denote by $\mathcal{T}(S)$ the suffix tree of $S \$$. $\mathcal{T}(S)$ has exactly $n+1$ leaves, each labeled by a position from $\{1, \ldots, n+1\}$, denoted $\ln (v)$. For node $v$ in $\mathcal{T}(S), L(v)$ denotes the string represented by $v$, i.e., the concatenation of edge labels on the path from the root to $v$. We denote by $t d(v)$ the treedepth of node $v$ and by $\operatorname{sd}(v)=|L(v)|$ its stringdepth. For internal node $v$, parent $(v)$ denotes $v$ 's parent and for character $c \in \Sigma$, $\operatorname{child}(v, c)$ denotes the child of $v$ reached by following the edge whose 
label starts with $c$ (if it exists). Given a node $u$ with parent $v$, a locus is a pair $(u, t)$ s.t. $s d(v)<t \leq s d(u)$. Let $[i, j]$ be the label of edge $(v, u)$ and $k=t-s d(v)$. We define $L(u, t)$ as the string $L(v) \cdot S[i, i+k-1]$, the substring represented by locus $(u, t)$. The one-to-one correspondence between loci of $\mathcal{T}(S)$ and substrings of $S \$$ allows us to define, for a substring $T$ of $S$, the locus of $T, \operatorname{loc}(T)=\operatorname{loc}(T, \mathcal{T}(S))$ as the unique locus $(u, t)$ in $\mathcal{T}(S)$ with the property that $L(u, t)=T$. Given $\operatorname{loc}(T)=(u, t)$, the set of occurrences of $T$ is the set $\{\ln (v) \mid v$ is leaf in the subtree rooted in $u\}$. Let $u$ be a node and $L(u)=c T$, where $c \in \Sigma$ and $T \in \Sigma^{*}$. The suffix link of $u$ is defined as $\operatorname{sink}(u)=\operatorname{loc}(T)$. We also define (implicit) suffix links for loci: for locus $(u, t)$ with $L(u, t)=c T$, define $\operatorname{slink}(u, t)=\operatorname{loc}(T)$. See Figure 2 .

Given a suffix tree $\mathcal{T}(S)$ with $k$ nodes, and a node $u$ of $\mathcal{T}(S)$, let $r$ be the rank of the node $u$ in the breath-first search traversal of the tree. We define the reverse index BFS of $u$ as $i B F S(u)=k-r$.

A maximum-oriented indexed priority queue [29, Sec. 2.4] denoted by $I P Q$, is a data structure that collects a set of $m$ items with keys $k_{1}, \ldots, k_{m}$ respectively, and provides operations: $\operatorname{insert}(i, k)$ (insert the element at index $i$ with key $k_{i}=k$ ); $\operatorname{demote}(i, k)$ (decreases the value of the key $k_{i}$, associated with $i$, to $\left.k \leq k_{i}\right) ;(i, k) \leftarrow \max ()$ (returns the index $i$ and the value $k$ of the item with maximum key $k_{i}$, breaking ties by index); $k \leftarrow \operatorname{keyOf}(i)$ (returns the value of the key $k_{i}$ associated with index $i$ ). Operations insert and demote run in $\mathcal{O}(\log (m))$ time, while the operations max, keyOf and isEmpty are performed in $\mathcal{O}(1)$ time. We also use a function $b \leftarrow$ allNegative () that returns true if all key values are negative, and false otherwise. We use the IPQ to store keys associated to nodes $u$ of a suffix tree $\mathcal{T}(S)$ using $i B F S(u)$ as index. For ease of presentation, in slight abuse of notation, we will use $u$ and $i B F S(u)$ interchangeably.

\section{Pattern discovery in colored strings using the suffix tree}

Our main tool will be the suffix tree of the reverse string, $\mathcal{T}=\mathcal{T}\left(S^{\text {rev }}\right)$. Note that loci in $\mathcal{T}$ correspond to ending positions of substrings of $S$ : given a locus $(u, t)$ of $\mathcal{T}$, let $U=L(u, t)^{\text {rev }}$. Then $U$ is a substring of $S$, and its occurrences are exactly the positions $i-|S|+1$, where $i=n-\ln (v)+1$ for some leaf $v$ in the subtree rooted in $u$. In the next lemma we show how to identify $(y, d)$-unique substrings of $S$ with $\mathcal{T}$.

- Lemma 3. Let $U$ be a substring of $S, \mathcal{T}=\mathcal{T}\left(S^{\text {rev }}\right)$, and $(u, t)=\operatorname{loc}\left(U^{\text {rev }}, \mathcal{T}\right)$. Then $U$ is y-unique with delay $d$ in $S$ if and only if for all leaves $v$ in the subtree rooted in $u$, $S^{r e v}[\ln (v)-d]$ is colored $y$ under $f^{r e v}$. In particular, $U$ is $y$-unique with delay 0 in $S$ if and only if all leaves in the subtree rooted in $u$ are colored $y$ under $f^{r e v}$.

In the following, we will refer to a node $u$ of $\mathcal{T}$ as $(y, d)$-unique if $L(u)^{\text {rev }}$ is a $(y, d)$-unique substring of $S$. We can now state the following corollary:

Corollary 4. Let $U$ be a substring of $S, \mathcal{T}=\mathcal{T}\left(S^{\text {rev }}\right)$, and $(u, t)=\operatorname{loc}\left(U^{\text {rev }}, \mathcal{T}\right)$ s.t. $u$ is an inner node of $\mathcal{T}(S)$. Then $U$ is $(y, d)$-unique in $S$ iff all children of $u$ are $(y, d)$-unique.

\subsection{Finding all $(y, d)$-unique substrings}

Our first algorithm Algo1 uses the suffix tree $\mathcal{T}$ of the reverse string to identify all $(y, d)$ unique substrings of $S$, not only the minimal ones, for fixed $y$ and $d$. It marks the $(y, d)$-unique nodes of $\mathcal{T}$ in a postorder traversal of the tree. Note that if $i>n-d$, then $i$ cannot be $(y, d)$-good, because the position in which we would expect a $y$ lies beyond the end of string

$S$. These positions are treated as if they were $(y, d)$-good (see Def. 1$)$.

The function $g(u): V(\mathcal{T}) \rightarrow\{0,1\}$ is defined as follows: 
- for a leaf $u$ with leaf number $\ln (u)=i: g(u)= \begin{cases}1 & \text { if either } i \leq d \text { or } f(i-d)=y \\ 0 & \text { otherwise }\end{cases}$ - for an inner node $u: g(u)= \begin{cases}y & \text { if } g(v)=1 \text { for all children } v \text { of } u \\ 0 & \text { otherwise }\end{cases}$

The algorithm computes $g(u)$ for every node $u$ in a bottom-up fashion. If $g(u)=1$, in addition it outputs all strings represented along the incoming edge of $u$, except for substrings

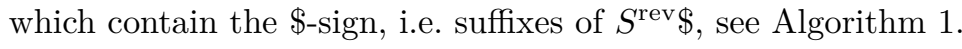

\section{Algorithm 1 AlgO1.}

input : A colored string $S$, the suffix tree $\mathcal{T}$ of $S^{\text {rev }}$, and $y \in \Gamma$.

output: All pairs $(T, d)$ such that $T$ is a $(y, d)$-unique substring of $S$.

1 for $d \leftarrow 0$ to $n$ do

$2 \leftarrow \operatorname{Unique}($ root, $y, d)$

3 procedure:

$4 \quad$ Unique $(u, y, d)$

5 if $u$ is a leaf then

$i \leftarrow \ln (u)$

if $i \leq d$ or $f^{r e v}(i-d)=y$ then

$\bar{g}(u) \leftarrow 1$

else

$g(u) \leftarrow 0$

else

$\left\lfloor g(u) \leftarrow \wedge_{v}\right.$ child of $u \operatorname{UniQue}(v, y, d)$

3 if $g(u)=1$ then

14 if $u$ is a leaf then // do not output $\$$-substrings

$15 \quad\left\lfloor\right.$ output $L(u, t)^{\mathrm{rev}}$ for every $t=\operatorname{sd}(\operatorname{parent}(u))+1, \ldots, \operatorname{sd}(u)-1$

16 else

else

output $\left(L(u, t)^{\mathrm{rev}}, d\right)$ for every $t=\operatorname{sd}(\operatorname{parent}(u))+1, \ldots, s d(u)$

18 return $g(u)$

Analysis: For fixed $d$, computing $g$ takes amortized $\mathcal{O}(n)$ time over the whole tree, since computing $g(u)$ is linear in the number of children of $u$, and therefore, charging the check whether for a child $v, g(v)=1$, to the child node, we get constant time per node. So, for fixed $d$, we have $\mathcal{O}(n+K)=\mathcal{O}\left(n^{2}\right)$ time, where $K$ is the number of $(y, d)$-unique substrings. Altogether, for $d=0, \ldots, n$, the algorithm takes $\mathcal{O}\left(n^{3}\right)$ time.

In the running example (Fig. 2), for color $y$ and delay $d=3$, the leaf nodes $9,2,7,1$, and 3 are marked with 1 , thus the only inner node $u$ which gets $g(u)=1$ is the parent of leaves number 9,2,7. Algo 1 outputs baca, cbaca, acbaca, cacbaca, acacbaca, cacacbaca, acacacbaca, caca, acaca, ca, aca, ab, cab, acab, bacab, cbacab, acbacab, cacbacab, acacbacab, cacacbacab, bac, cbac, acbac, cacbac, acacbac, cacacbac, acacacbac.

Remark: Note some of these substrings do not occur even once in a position such that the last character is followed by a $y$ with delay $d=3$. For instance, the only occurrence of the substring bac in $S$ is at position 7, so we would expect to see color $y$ at position $9+3=12$, but the string $S$ ends at position 11. We treat this and similar questions in Section 5 .

\subsection{Outputting only minimally $(y, d)$-unique substrings}

We next modify Algorithm ALGO1 to output only minimally $(y, d)$-unique substrings. In the suffix tree $\mathcal{T}$ of $S^{\text {rev }}$, minimality translates into conditions on the parent node and on the 
suffix link parent node (equivalently: the suffix link) in $\mathcal{T}$. We first need another definition:

- Definition 5 (Left-minimal nodes, left-minimal labels). Let $u$ be a node of $\mathcal{T}=\mathcal{T}\left(S^{\text {rev }}\right)$, different from the root, and let $v=\operatorname{parent}(u)$. We call $u$ left-minimal for $(y, d)$ if $u$ is $(y, d)$-unique but $v$ is not and the label of the edge $(v, u)$ is not equal to $\$$. If $u$ is $(y, d)$-unique and left-minimal, then we can define Left-min $(u)=x_{1} \cdot L(v)^{\text {rev }}$, the left-minimal $(y, d)$-unique substring of $S$ associated to $u$, where $x=x_{1} \cdots x_{k} \in \Sigma^{+}$is the label of edge $(v, u)$.

In our running example, let $u=\operatorname{loc}(\mathcal{T}$, aca $)$. Then $u$ is left-minimal, since it is $(y, 3)$ unique but its parent is not. Its left-minimal label is Left-min $(u)=$ ca. See Fig. 2.

It is easy to modify Algorithm 1 to output only left-minimal substrings: Whenever for an inner node $u$ we get $g(u)=0$, then for every child $v$ of $u$ with $g(v)=1$, we output Left-min $(v)$ (if defined). This means replacing lines 13 to 17 in Algorithm 1 (details left to the reader).

In the example, we would now output, for color $y$ and $d=3$, the left-minimal substrings $\mathrm{ca}, \mathrm{ab}, \mathrm{bac}$. However, we are interested in substrings which are both left- and right-minimal. For right-minimality, Obs. 1 part (3) tells us that we need to check whether the string without the last character is $(y, d+1)$-unique. In $\mathcal{T}$, this translates to checking the suffix link of the locus of the left-minimal substring $\operatorname{Left}-\min (u)$.

Proposition 6. Let $u$ be an inner node of $\mathcal{T}=\mathcal{T}\left(S^{\text {rev }}\right)$, different from the root, s.t. $L(u)^{\text {rev }}$ is $(y, d)$-unique in $S$. Let $v=\operatorname{parent}(u)$, and $x_{1}$ be the first character on the edge $(v, u)$. Further, let $t=s d(v)+1$, and $\left(u^{\prime}, t^{\prime}\right)=\operatorname{slink}(u, t)$. Then the substring $U=x_{1} \cdot L(v)^{\text {rev }}$ is minimally $(y, d)$-unique in $S$ iff $v$ is not $(y, d)$-unique and $u^{\prime}$ is not $(y, d+1)$-unique.

We can use Prop. 6 as follows. Once a left-minimal $(y, d)$-unique node $u$ has been found, check whether $u^{\prime}$ is $(y, d+1)$-unique, where $u^{\prime}$ is the node below the locus $\operatorname{sink}(u, \operatorname{sd}(\operatorname{parent}(u))+$ $1)$. It is easy to find node $u^{\prime}$ by noting that $u^{\prime}=\operatorname{child}\left(\operatorname{slink}(\operatorname{parent}(u)), x_{1}\right)$, where $x_{1}$ is the first character of the edge label leading to $u$. To know whether $u^{\prime}$ is $(y, d+1)$-unique, we process the distances $d$ in descending order, from $d=n$ down to $d=0$. At the end of the iteration for $d$, we retain the information, keeping a flag on every node $u$ which is $(y, d)$-unique. See Algorithm 2.

In the running example, we know from the previous round for $d=4$ that the only nodes that are $(y, 4)$-unique are the leaves number $4,2,1,10,3$, and 8 . We can now deduce that the substring ca is right-minimal, because $u=\operatorname{loc}(\mathrm{ca})$ is not $(y, 4)$-unique, and $\operatorname{slink}\left(\operatorname{loc}\left(\mathcal{T}, \mathrm{ca}^{\mathrm{rev}}\right)\right)=(u, 1)$. Looking at the string $S$ we see that ca is indeed right-minimal, since $\mathrm{c}$ is not $(y, 3)$-unique: it has an occurrence, in position 6 , which is not followed by a $y$ but by an $x$ at position $10=6+4$ (delay 4 ). Similarly, the other two left-minimal substrings $\mathrm{ab}$ and bac are also right-minimal, because their respective suffix links are not $(y, 4)$-unique.

For fixed $d$, the time spent on each leaf is constant (lines 5 to 10 in Algo2); we charge the check of $g(v)$ in line 12 to the child $v$, as well the work in lines 14 to 18 (computing $\operatorname{Left-\operatorname {min}}(v)$ and checking the flag on $v^{\prime}$ from the previous round); these are all constant-time operations, and so we have $\mathcal{O}(n)$ time for fixed $d$ and $\mathcal{O}\left(n^{2}\right)$ in total.

\section{$4 \quad$ Skipping Algorithm}

We now focus on discovering minimal $(y, d)$-unique substrings. As before, we build $\mathcal{T}\left(S^{\text {rev }}\right)$ and traverse it, discovering all left-minimal $(y, d)$-unique substrings as we go, reporting only those that are minimal. Thus, by Proposition 6 , we have to discover all left-minimal $(y, d+1)$-unique substrings before discovering left-minimal $(y, d)$-unique ones.

To this end, fixing $\ell$, for each node $u$ of $\mathcal{T}\left(S^{\text {rev }}\right)$, we determine the largest delay $d$ smaller than $\ell$ such that $L(u)^{\text {rev }}$ can be $(y, d)$-unique, denoted by $h(u, \ell)$. We consider four cases: 
Algorithm 2 AlgO2.

input : a colored string $S$, the suffix tree $\mathcal{T}$ of $S^{\text {rev }}$ with suffix links, and $y \in \Gamma$.

output: all pairs $(T, d)$ such that $T$ is a minimally $(y, d)$-unique substring of $S$.

1 for $d \leftarrow n$ downto 0 do

$2 \mid \operatorname{MinUnique}($ root, $y, d)$

3 procedure:

$4 \quad$ MinUnique $(u, y, d)$

5 if $u$ is a leaf then

$6 \quad i \leftarrow \ln (u)$

$7 \quad$ if $i \leq d$ or $f^{\text {rev }}(i-d)=y$ then

$8 \quad\lfloor g(u) \leftarrow 1$

$9 \quad$ else

$10 \quad\lfloor g(u) \leftarrow 0$

11 else

$12 \mid g(u) \leftarrow \wedge_{v}$ child of $u \operatorname{MinUnique}(v, y, d)$

14 for each child $v$ with $g(v)=1$ do

// outputting minimal substrings for children

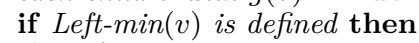

$\left(v^{\prime}, t\right) \leftarrow \operatorname{sink}(v, \operatorname{sd}(u)+1)$

if $v^{\prime}$ is not $(y, d+1)$-unique then $\quad / /$ flag from previous round

L output $(\operatorname{Left-min}(v), d)$

19 return $g(u)$

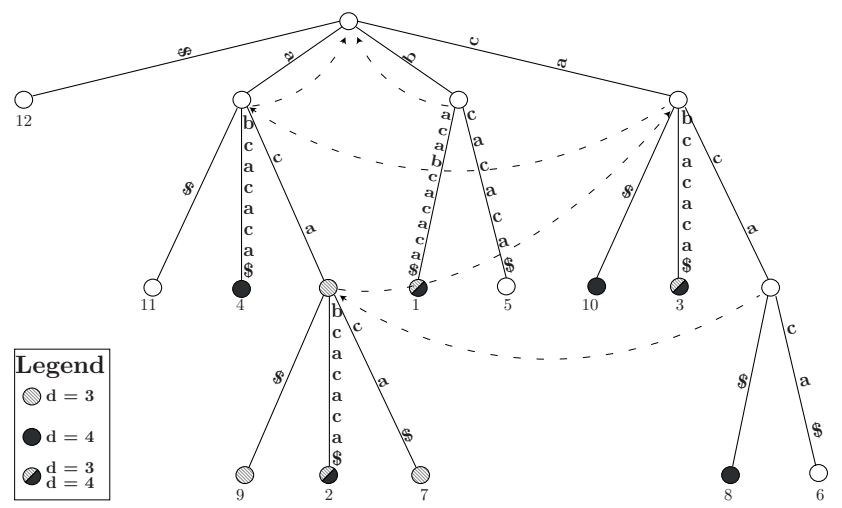

Figure 2 The suffix tree $\mathcal{T}$ of the reverse string $S^{\text {rev }}=$ bacabcacaca, where $S=$ acacacbacab (our running example). For clarity, the edges carry the label itself rather than a pair of pointers into the string. Suffix links are drawn as dotted directed edges. The nodes are colored according to function $g$ for the character $y$, for $d=3$ (dashed) and for $d=4$ (solid). 
- If $u$ is a leaf, then $L(u)^{\text {rev }}$ is the $j$-prefix of $S$, where $j=n-\ln (u)+1=|L(u)|$

- If $\ln (u)<\ell$, then $j+\ell-1>n$ thus $L(u)^{\text {rev }}$ is $(y, \ell-1)$-unique since the position of the color is beyond the end of the string, thus $h(u, \ell)=\ell-1$.

- If $\ln (u) \geq \ell$ and there exists an $i<\ell$ such that $f(j+i)=y$, then the highest possible value $d<\ell$ such that $L(u)^{\text {rev }}$ is $(y, d)$-unique is given by the position of the furthest occurrence of $y$ within a distance of $\ell-1$ from $j$, thus $h(u, \ell)=\max \{i<\ell \mid f(j+i)=y\}$.

- Otherwise, if such $i$ does not exists, we set $h(u, \ell)=-1$.

- If $u$ is an internal node of $\mathcal{T}\left(S^{\mathrm{rev}}\right)$, then let $k=\min \{h(v, \ell) \mid v$ child of $u\}$, since it is not possible that $L(u)^{\text {rev }}$ is $\left(y, d^{\prime}\right)$-unique, for any $k<d^{\prime}<\ell$, thus $h(u, \ell)=k$.

When $u$ is an inner node, in general, we do not know if $L(u)^{\text {rev }}$ is $(y, d)$-unique for $d=h(u, \ell)$, unless for all nodes $v$ in the subtree rooted at $u$, there exists an $\ell_{v}$ such that $h(u, \ell)<\ell_{v} \leq \ell$ and $h\left(v, \ell_{v}\right)=h(u, \ell)$. This is true if $h(v, d+1)=h(u, \ell)$ for all $v$.

- Lemma 7. Let $u$ be a node of $\mathcal{T}\left(S^{r e v}\right)$, fix $d, h(u, d+1)=d$ if and only if $u$ is $(y, d)$-unique.

To evaluate $h(u, \ell)=\max \{i<\ell \mid f(j+i)=y\}$ when $u$ is a leaf and such $i$ exists, we define a bitvector $b_{y}[1,2 n]$ such that $b_{y}[i]=1$ only if $f(i)=y$ or $i>n$. We preprocess $b_{y}$ for $\mathcal{O}(1)$-time rank and select queries [5]. Given node $u$ with $\ln (u) \geq \ell$, let $j=n-\ln (u)+1$. We have that $h(u, \ell)=\max \left\{\operatorname{select}\left(b_{y}, \operatorname{rank}\left(b_{y}, j+\ell\right)\right)-j,-1\right\}$.

We use the $h(u, \ell)$ function in the following way, during the discovery process of all $(y, d)$-unique substrings of $S$, provided that we have already discovered all $(y, d+1)$-unique substrings of $S$. Let $\ell=d+1$, for all nodes $u$ of $\mathcal{T}\left(S^{\text {rev }}\right)$ we store the values $h(u, \ell)$. We discover the minimally $(y, d)$-unique substrings of $S$, finding all nodes $u$ such that $h(u, \ell)=d$. Among those, the nodes that are also left-minimal are those nodes $u$ such that, $h($ parent $(u), \ell)<d$. We then check if $u$ is also right-minimal by checking if its suffix-link parent is $(y, d+1)$-unique, as in Algorithm 2.

The key idea of the skipping algorithm is to keep the values $h(u, \ell)$ updated during the process. Let $H(u)$ be the array that, at the beginning of the discovery of all $(y, d)$-unique substrings of $S$, stores the values $h(u, \ell)$. We want to keep the array $H$ updated in a way such that, after we discovered all $(y, d)$-unique substrings of $S$, for all nodes $u, H(u)=h(u, \ell-1)$. Thus, once we discover that a node $u$ is left-minimal $(y, d)$-unique, we update the value of $H(u)=h(u, \ell-1)$. We then update the following values:

- for all nodes $v$ in the subtree rooted in $u$, we update the values $H(u)=h(u, \ell-1)$.

- for all nodes $p$ ancestors of $u$, we update the values $H(p)=\min (H(p), h(u, \ell-1))$

- Lemma 8. Given $\mathcal{T}\left(S^{\text {rev }}\right)$, fix $d$, for all nodes u of $\mathcal{T}\left(S^{\text {rev }}\right)$, let $H(u)=h(u, d+1)$. If for all nodes $u$ such that $H(u)=d$ we $(i)$ set $H(u)=h(u, d)$, and (ii) for all ancestors $p$ of $u$, set $H(p)=\min \{H(p), h(u, d)\}$, then, for all nodes $u$ of $\mathcal{T}\left(S^{r e v}\right), H(u)=h(u, d)$.

In order to efficiently find all nodes $u$ such that $h(u, \ell)=d$ and $h($ parent $(u), \ell)<d$, we use a maximum-oriented indexed priority queue, storing the values of $H(u)$ as keys and $i B F S(u)$ as index. Under this condition, if two nodes have the same key value, then parents have higher priority than their children in $I P Q$. We keep the priority queue updated using a demote operation while we discover left-minimal nodes and we update the values of the array $H$ stored as keys of $I P Q$. Algorithm 3 shows how to compute $h(u, \ell)$ for a given node $u$, and how we update the values of the keys in the IPQ for all children $v$ of $u$.

The skipping algorithm (see Algorithm 4), initializes priority queue $I P Q$ by inserting all nodes of $\mathcal{T}\left(S^{\text {rev }}\right)$ with key $n+1$. We then repeat the following until there exists a node with non-negative key: extract the max element $(u, \ell)$ of $I P Q$, decide whether it has to be 
Algorithm $3 h(u, d)$.

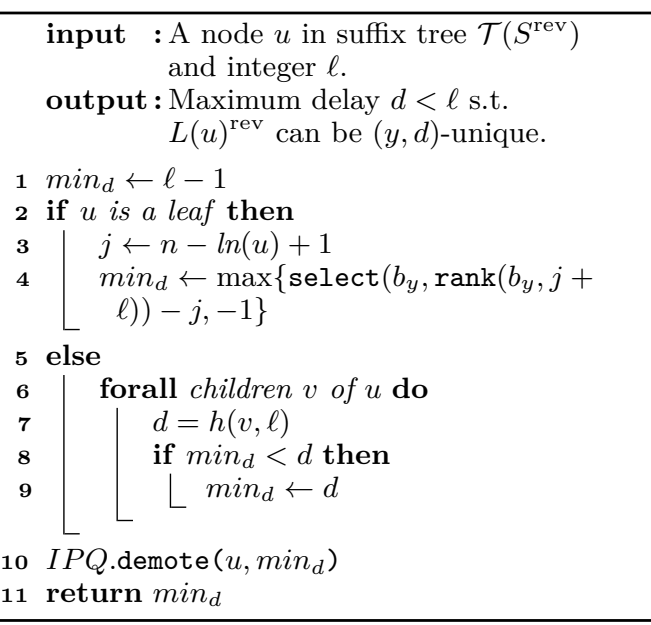

Algorithm 4 SKIPPING.

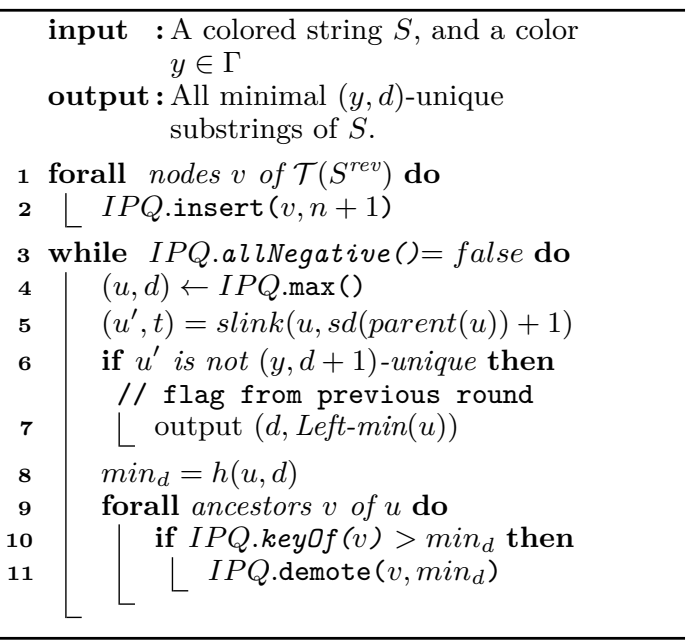

reported, i.e. if it is right-minimal; apply Algorithm 3 to update the key values of all nodes in the subtree at $u$ and then update the values of the keys of all ancestors of $u$.

For all nodes $u$ in $\mathcal{T}\left(S^{\text {rev }}\right)$, the key value associated to $u$ in $I P Q$ is initially $n+1$. Each time Algorithm 4 and Algorithm 3 visit a node, the key value of $u$ in $I P Q$ is decreased (via demote ()) until it becomes negative. Thus, for each node we perform at most $n+1$ demote() operations. Since the number of nodes in $\mathcal{T}\left(S^{\text {rev }}\right)$ is linear in $n$, Algorithm 4 runs in $\mathcal{O}\left(n^{2} \log (n)\right)$ time.

\section{Output restrictions and algorithm improvement}

We now discuss some practically-minded output restrictions. These could be implemented as a filter to the output, thus discarding some solutions, but when considered as part of the problem, they lead to an improvement for the skipping algorithm.

Note that our definition of $(y, d)$-unique allows that a substring occurs only once, or that none of its occurrences is followed by a $y$ with delay $d$, because they are all close to the end of string. We restrict our attention to $(y, d)$-unique substrings with at least two occurrences followed by $y$ with delay $d$. Given a colored string $S$, let $T$ be minimally $(y, d)$-unique. Now we report $(T, d)$ if and only if the following holds: 1$)$ there are at least two occurrences of $T$ in $S ; 2)$ let $i$ be the second smallest occurrence of $T$ in $S$, then $i+|T|-1+d \leq n$.

A substring $T$ that satisfies the above conditions is called real type minimally $(y, d)$-unique substring. In order to satisfy those conditions, it is enough to perform the output operations at line 7 of Algorithm 4 and at line 18 of Algorithm 2 if the node $u$ is not a leaf and the value of the second greatest suffix of $S^{\text {rev }}$ in the subtree rooted in $u$ is greater than or equal to $d$. Since each node $u$ in the suffix tree $\mathcal{T}\left(S^{\mathrm{rev}}\right)$, corresponds to an interval $[i, j]$ of the suffix array of $S^{\text {rev }}$, we can find the second greatest suffix using a range maximum query $r M q$ data structure [11] built on the suffix array of $S^{\text {rev }}$. Then, the second greatest suffix can be found in $\mathcal{O}(1)$ time, using $2 n+o(n)$ bits of extra space.

The $h(u, \ell)$ function is used in Algorithm 4 in order to find left-minimal nodes in the suffix tree. If we consider the output restrictions as part of the problem, then we do not have to report minimally $(y, d)$-unique substrings that occur only once, i.e., leaves in $\mathcal{T}\left(S^{\text {rev }}\right)$. Then, for all nodes $u$ such that all children of $u$ are leaves, we can directly compute the 
Table 1 Real-world datasets used in the experiments. In columns 1 and 2, we report names and descriptions of the hardware designs used to generate the simulation traces. In columns 3 and 4 , we give the number of primary inputs resp. of primary outputs. In column 5 we report the length of the simulation trace, and in columns 6 and 7 the size of the alphabet and the number of colors, respectively. For each design we fixed a color $y$, and report in col. 8 number $n_{y}$ of $y$ characters.

\begin{tabular}{llrrrrrr}
\hline Design & Description & PIs & POs & $n$ & $\sigma$ & $\gamma$ & $n_{y}$ \\
\hline b03 & Resource arbiter [6] & 6 & 4 & 100000 & 17 & 5 & 3210 \\
b06 & Interrupt handler [6] & 4 & 6 & 100000 & 5 & 4 & 44259 \\
s386 & Shynthetized controller [3] & 9 & 7 & 100000 & 129 & 2 & 8290 \\
camellia & Symmetric key block cypher [26] & 262 & 131 & 103615 & 70 & 224 & 2292 \\
serial & Serial data transmitter & 11 & 2 & 100000 & 118 & 2 & 16353 \\
master & Wishbone bus master [26] & 134 & 135 & 100000 & 417 & 80 & 759 \\
\hline
\end{tabular}

highest value of $d<\ell$ such that $L(u)^{\text {rev }}$ is $(y, d)$-unique. This leads to the definition of the fast_h $(u, \ell)$ function for a node $u$ of $\mathcal{T}\left(S^{\text {rev }}\right)$. The function fast_h $h(u, \ell)$ is defined similarly to the function $h(u, \ell)$ with the additional following case: If all children of $u$ are leaves, we can directly compute the highest value of $d<\ell$ such that $L(u)^{\text {rev }}$ is $(y, \ell)$-unique as the largest value $d<\ell$ such that, for each child $v$ of $u, h(v, d+1)=d$. In other words, we want the largest $d<\ell$ such that all children of $v$ are $(y, d)$-unique.

\section{Experimental results}

We implemented the algorithms ${ }^{1}$ and measured their performance on randomly generated datasets and real-world datasets. Experiments were performed on a $3.4 \mathrm{GHz}$ Intel Core i7-6700 CPU with $8 \mathrm{MiB}$ L3 cache and $16 \mathrm{GiB}$ of DDR4 RAM running Ubuntu 16.04 (64bit, kernel 4.4.0). The compiler was g++ version 5.4.0 with -03 -DNDEBUG -funroll-loops -msse4.2 options. Runtimes were recorded with the $\mathrm{C}++11$ high_resolution_clock facility.

We used two different datasets, one consisting of randomly generated data and one consisting of real-world data. Randomly generated data varied string length $n=100,1000$, 10000,100000 , alphabet size $\sigma=2,4,8,16,32$, and number of colors $\gamma=2,4,8,16,32$. Strings were generated one character (and color) at a time, i.e. fixing $\sigma$ and $\gamma$, the string of length $n=1000$ is a prefix of the string $n=10000$. Characters and colors follow a uniform distribution. We report only on experiments for $n=1000,10000,100000, \sigma=2,8,32$, color number $\gamma=2,8,32$, and seed 0 , which are representative of the trend we observed for all settings. The real-world data is the result of a simulation on a set of established benchmarks in embedded systems verification $[3,6,26]$, reported in Table 1 . The benchmarks are descriptions of hardware design at the register-transfer level of abstraction.

We compared implementations base (the baseline, Algorithm 2); skip (the skipping algorithm in Algorithm 4, using the $h$ function in Algorithm 3); and real (as skip, but using the fast_h function). All algorithms report minimally $(y, d)$-unique substrings only if they are real type. We used the sdsl-lite library [17] for compressed suffix trees and supporting data structures.

We performed all experiments five times and report the average. Results are reported in Figure 3 and Table 2. Figure 3 shows the results for base, skip and real on the random data set. We observe how the algorithms scale with respect to increasing the number of colors, which has the effect of reducing the number of $y$-colored characters; increasing alphabet size;

1 Available online at: https://github.com/maxrossi91/colored-strings-miner. 
and increasing the length of the text. In summary, for all algorithms: running time decreases with increasing number of colors and increases with alphabet size. We observe a quadratic dependence on $n$ in line with our asymptotic analysis.
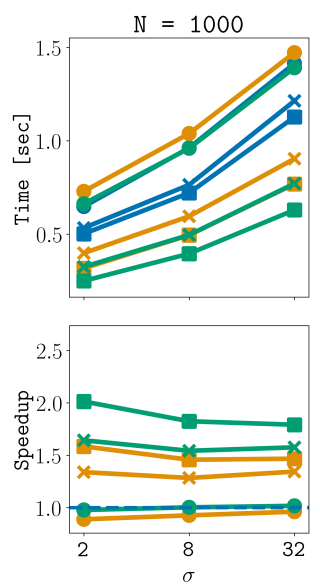
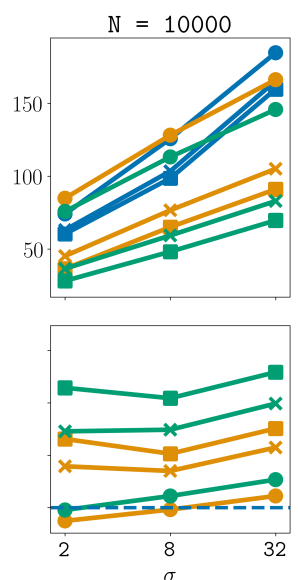
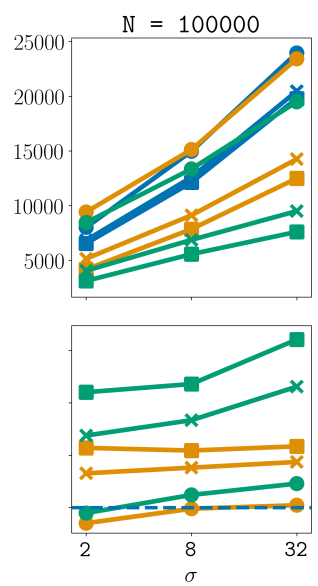

Legend

- base $-\gamma=2$

$\times$ base $-\gamma=8$

base $-\gamma=32$

- $\operatorname{skip}-\gamma=2$

$\times \quad$ skip $-\gamma=8$

skip $-\gamma=32$

- real $-\gamma=2$

$\times$ real $-\gamma=8$

- real $-\gamma=32$

Figure 3 Results of the execution of algorithms base (color blue), skip (color orange) and real (color green) over the randomly generated data for $N=10^{3}, 10^{4}$, and $10^{5}$. The $x$ axis represents the values of $\sigma=\{2,8,32\}$, and the different markers represents the values of $\gamma=\{2,8,32\}$ (resp. circles, crosses, and boxes). The three plots in the first row report execution times. The plots in the second row report speedups of skip and real with respect to algorithm base represented as the dashed blue line at constant 1.0.

Figure 3 shows that the skip algorithm is almost always faster than the base algorithm, and that the average speedup is 1.30 , with a maximum of 1.75 . Moreover, we have that the the real algorithm is almost always faster than the skip algorithm, and the average speedup is 1.25, with a maximum of 1.64. Finally, the average speedup between real and base is 1.65 , with a maximum of 2.60 in the case of $N=100000, \sigma=32$ and $\gamma=32$.

Table 2 shows the results for base, skip and real algorithms on the real dataset. Here, we observe a similar trend to the random data, but the speedup of real with respect to base is much higher -3.40 on average, with a maximum of 11.88 on the master device. However, on three of the six datasets, base is faster than skip, and faster than real on one.

\section{Conclusion}

We studied pattern discovery problems on colored strings motivated by applications in embedded system verification. To the best of our knowledge this is the first principled

Table 2 Results of the execution of algorithms base, skip and real over the real-world dataset. The first column reports the name of the design from which the simulation trace is retrieved.

\begin{tabular}{lcccccc}
\hline & \multicolumn{3}{c}{ Execution Time $(\mathrm{sec})$} & \multicolumn{3}{c}{ Speedup (ratio) } \\
Design & base & skip & real & base $/$ skip & skip / real & base $/$ real \\
\hline b03 & 2258.94 & 3925.08 & 3149.46 & 0.58 & 1.25 & 0.72 \\
b06 & 3575.78 & 5463.50 & 4511.97 & 0.65 & 1.21 & 0.79 \\
s386 & 3285.55 & 5347.90 & 3719.14 & 0.61 & $\mathbf{1 . 4 4}$ & 0.88 \\
camellia & 3015.91 & 1098.77 & 1071.75 & 2.74 & 1.03 & 2.81 \\
serial & 3325.84 & 989.98 & 1003.72 & 3.36 & 0.99 & 3.31 \\
master & 3365.56 & 284.19 & 283.24 & $\mathbf{1 1 . 8 4}$ & 1.00 & $\mathbf{1 1 . 8 8}$ \\
\hline
\end{tabular}


algorithmic treatment of these problems. Our fastest algorithm stores, during the discovery process, for each distinct substring the next delay value which is $(y, d)$-unique, using a priority queue to find these values and to identify minimally $(y, d)$-unique substrings. The algorithm is especially fast on real-world instances. Under a variant of the minimality condition oriented toward real-world instances the algorithm becomes even faster. We are currently working with colleagues in embedded systems to integrate these algorithms into their analysis workflows.

\section{- References}

1 Rakesh Agrawal and Ramakrishnan Srikant. Fast algorithms for mining association rules. In Proc. 20th International Conference on Very Large Data Bases (VLDB), volume 1215, pages 487-499. Morgan Kaufmann, 1994.

2 Rakesh Agrawal and Ramakrishnan Srikant. Mining sequential patterns. In Proc. Eleventh International Conference on Data Engineering (ICDE), volume 95, pages 3-14. IEEE Computer Society, 1995.

3 Franc Brglez, David Bryan, and Krzysztof Kozminski. Combinational profiles of sequential benchmark circuits. In Proc. 1989 IEEE international symposium on circuits and systems (ISACS), volume 3, pages 1929-1934. IEEE, 1989.

4 Chung-Wen Cho, Ying Zheng, Yi-Hung Wu, and Arbee LP Chen. A tree-based approach for event prediction using episode rules over event streams. In Proc. International Conference on Database and Expert Systems Applications (DEXA 2008), volume 5181 of LNCS, pages 225-240. Springer, 2008.

5 David Clark. Compact PAT trees. PhD thesis, University of Waterloo, 1997.

6 Fulvio Corno, Matteo Sonza Reorda, and Giovanni Squillero. Rt-level itc'99 benchmarks and first atpg results. IEEE Design \& Test of computers, 17(3):44-53, 2000.

7 Alessandro Danese, Nicolò Dalla Riva, and Graziano Pravadelli. A-team: Automatic templatebased assertion miner. In Proc. 2017 54th ACM/EDAC/IEEE Design Automation Conference (DAC), pages 1-6. IEEE, 2017.

8 Alessandro Danese, Tara Ghasempouri, and Graziano Pravadelli. Automatic extraction of assertions from execution traces of behavioural models. In Proc. 2015 Design, Automation $\&$ Test in Europe Conference 86 Exhibition (DATE), pages 67-72. IEEE, 2015.

9 Jasbir Dhaliwal, Simon J Puglisi, and Andrew Turpin. Practical efficient string mining. IEEE Transactions on Knowledge and Data Engineering, 24(4):735-744, 2010.

10 Lina Fahed, Armelle Brun, and Anne Boyer. DEER: Distant and Essential Episode Rules for early prediction. Expert Systems with Applications, 93:283-298, 2018.

11 Johannes Fischer and Volker Heun. Space-efficient preprocessing schemes for range minimum queries on static arrays. SIAM J. Comput., 40(2):465-492, 2011.

12 Johannes Fischer, Volker Heun, and Stefan Kramer. Fast frequent string mining using suffix arrays. In Proc. Fifth IEEE International Conference on Data Mining (ICDM 2005), pages 609-612. IEEE, 2005.

13 Johannes Fischer, Volker Heun, and Stefan Kramer. Optimal string mining under frequency constraints. In Proc. European Conference on Principles of Data Mining and Knowledge Discovery (PKDD 2006), volume 4213 of LNCS, pages 139-150. Springer, 2006.

14 Johannes Fischer, Veli Mäkinen, and Niki Välimäki. Space efficient string mining under frequency constraints. In Proc. Eighth IEEE International Conference on Data Mining (ICDM 2008), pages 193-202. IEEE, 2008.

15 Harry D Foster, Adam C Krolnik, and David J Lacey. Assertion-based design. Springer Science \& Business Media, 2004.

16 Philippe Fournier-Viger, Jerry Chun-Wei Lin, Rage Uday Kiran, Yun Sing Koh, and Rincy Thomas. A survey of sequential pattern mining. Data Science and Pattern Recognition, 1(1):54-77, 2017. 
17 Simon Gog, Timo Beller, Alistair Moffat, and Matthias Petri. From Theory to Practice: Plug and Play with Succinct Data Structures. In 13th International Symposium on Experimental Algorithms, (SEA 2014), volume 8504 of LNCS, pages 326-337. Springer, 2014.

18 Dan Gusfield. Algorithms on Strings, Trees, and Sequences : Computer Science and Computational Biology. Cambridge University Press, Cambridge, United Kingdom, 1997.

19 Koji Iwanuma, Ryuichi Ishihara, Yoh Takano, and Hidetomo Nabeshima. Extracting frequent subsequences from a single long data sequence a novel anti-monotonic measure and a simple on-line algorithm. In Proc. Fifth IEEE International Conference on Data Mining (ICDM 2005), pages 186-195. IEEE, 2005.

20 Xiaonan Ji, James Bailey, and Guozhu Dong. Mining minimal distinguishing subsequence patterns with gap constraints. Knowledge and Information Systems, 11(3):259-286, 2007.

21 Srivatsan Laxman, Vikram Tankasali, and Ryen W White. Stream prediction using a generative model based on frequent episodes in event sequences. In Proc. 14th ACM SIGKDD international conference on Knowledge discovery and data mining (KDD 2008), pages 453-461. ACM, 2008.

22 Lingyi Liu, David Sheridan, Viraj Athavale, and Shobha Vasudevan. Automatic generation of assertions from system level design using data mining. In Proc. Ninth ACM/IEEE International Conference on Formal Methods and Models for Codesign, pages 191-200. IEEE Computer Society, 2011.

23 Nizar R Mabroukeh and Christie I Ezeife. A taxonomy of sequential pattern mining algorithms. ACM Computing Surveys (CSUR), 43(1):3, 2010.

24 Veli Mäkinen, Djamal Belazzougui, Fabio Cunial, and Alexandru I. Tomescu. Genome-Scale Algorithm Design. CUP, 2015.

25 Heikki Mannila, Hannu Toivonen, and A Inkeri Verkamo. Discovery of frequent episodes in event sequences. Data mining and knowledge discovery, 1(3):259-289, 1997.

26 OpenCores. Available at https://opencores.org/. Accessed 05-03-2019.

27 Tinghai Pang, Lei Duan, Jesse Li-Ling, and Guozhu Dong. Mining Similarity-Aware Distinguishing Sequential Patterns from Biomedical Sequences. In Proc. Second International Conference on Data Science in Cyberspace (DSC 2017), pages 43-52. IEEE, 2017.

28 Jian Pei, Jiawei Han, and Wei Wang. Constraint-based sequential pattern mining: the pattern-growth methods. Journal of Intelligent Information Systems, 28(2):133-160, 2007.

29 Robert Sedgewick and Kevin Wayne. Algorithms. Addison-Wesley Professional, 2011.

30 Bill Smyth. Computing Patterns in Strings. Pearson Addison-Wesley, Essex, England, 2003.

31 Niko Välimäki and Simon J Puglisi. Distributed string mining for high-throughput sequencing data. In Proc. 12th International Workshop on Algorithms in Bioinformatics (WABI 2012), volume 7534 of LNCS, pages 441-452. Springer, 2012.

32 Shobha Vasudevan, David Sheridan, Sanjay Patel, David Tcheng, Bill Tuohy, and Daniel Johnson. Goldmine: Automatic assertion generation using data mining and static analysis. In Proc. 2010 Design, Automation 8 Test in Europe Conference 8 Exhibition (DATE 2010), pages 626-629. IEEE, 2010.

33 Xianming Wang, Lei Duan, Guozhu Dong, Zhonghua Yu, and Changjie Tang. Efficient mining of density-aware distinguishing sequential patterns with gap constraints. In International Conference on Database Systems for Advanced Applications (DASFAA 2014), volume 8421 of LNCS, pages 372-387. Springer, 2014.

34 Xindong $\mathrm{Wu}$, Xingquan Zhu, Yu He, and Abdullah N Arslan. PMBC: Pattern mining from biological sequences with wildcard constraints. Computers in Biology and Medicine, 43(5):481-492, 2013. 